\title{
Diagnostic investigation of breast magnetic resonance imaging in malignant and benign mass lesions
}

Masoumeh Gity ${ }^{1,2}$, Ali Arabkheradmand ${ }^{3}$, Elham Taheri ${ }^{1}$, Madjid Shakiba ${ }^{1}$

\author{
${ }^{1}$ Advanced Diagnostic and Interventional Radiology Research Center (ADIR), \\ Tehran University of Medical Sciences, Tehran, Iran \\ ${ }^{2}$ Department of Radiology, Medical Imaging Center, Tehran University of Medical \\ Sciences, Tehran, Iran \\ ${ }^{3}$ Department of Surgery, Cancer Institute, Tehran University of Medical Sciences, \\ Tehran, Iran
}

Submitted: 28 July 2016

Accepted: 1 August 2016

Arch Med Sci 2018; 14, 5: 1061-1069

DOI: https://doi.org/10.5114/aoms.2016.62281

Copyright @ 2016 Termedia \& Banach

\section{Abstract}

Introduction: Breast magnetic resonance imaging (BMRI) has been identified as a valuable modality in the diagnosis of breast cancer and monitoring the response to chemotherapy. The aim of this study was to evaluate the relative importance of different descriptors of breast masses in contrast-enhanced breast MRI.

Material and methods: In a database of pathologically proven breast lesions, in total 433 masses in 312 patients detected by contrast-enhanced breast MRI were selected. All images were assessed according to the MRI BI-RADS lexicon and those with significant positive MRI findings (BI-RADS categories 3, 4, 5) were enrolled in the study.

Results: Mean age of patients was $45.09 \pm 10.5$ years. The most frequent BI-RADS score was 4 (60.7\%), followed by 3 (27\%). Among the morphologic descriptors of the enhancing masses, the findings most strongly associated with malignancy included spiculated margin (60.6\%) and irregular shape $(38 \%)$. Considering the dynamic descriptors, a wash-out pattern in the time-intensity curve was the most powerful finding associated with malignancy (27.9\%). Among all breast MRI descriptors, the best odds ratio (OR) in association with malignancy was noted for speculated margin $(O R=10.2)$ followed by wash-out or plateau curves $(O R=6.1)$, size greater than $1 \mathrm{~cm}$ $(O R=4.3)$ and irregular shape $(O R=3.1)$.

Conclusions: It seems that morphologic descriptors of MRI BI-RADS for enhancing masses are quite specific, while dynamic descriptors of the masses are highly sensitive. Appropriate consideration and combination of different BI-RADS findings could help in better characterization of enhancing masses on breast MRI, lowering the rate of false positive reports and avoiding unnecessary biopsies.

Key words: breast, magnetic resonance imaging, mass, diagnosis, biopsy.

\section{Introduction}

Breast magnetic resonance imaging (BMRI) has been identified as a valuable modality in the diagnosis of breast cancer [1, 2], and monitoring the response to chemotherapy [3-5]. The sensitivity of breast MRI for diagnosing invasive breast cancer has been reported between $88 \%$ and $100 \%$, surpassing all other conventional modalities such as mammog-

\author{
Corresponding author: \\ Dr. Elham Taheri \\ Advanced Diagnostic \\ and Interventional Radiology \\ Research Center (ADIR) \\ Medical Imaging Center \\ Keshavarz Blvd. \\ Tehran, 1419733141, Iran \\ Phone: +98-912 6200762 \\ E-mail: elhamtaheri.md@ \\ gmail.com
}


raphy and ultrasound [6]. However, its specificity in discriminating malignant from benign lesions is highly varied, reported in the range of $35 \%$ to $90 \%[7,8]$. Different lesions found in breast MRI include enhancing masses, non-mass-like enhancing lesions and enhancing foci.

The MRI BI-RADS lexicon has been developed in order to obtain a standard system of reporting. This lexicon defines morphologic and kinetic descriptors for assessing breast lesions and finally scores the lesion in a range of BI-RADS categories from 0 to 6 . Although some paradigms for interpretation and scoring of the lesions in the $\mathrm{BI}$ RADS system have been proposed, the final scoring is somewhat subjective. As the assessment is based on different morphologic and dynamic criteria, a critical question is the relative importance of each descriptor in the final assessment of breast MRI which could optimize the accuracy of the MRI report. A review of the literature shows that there have been similar studies with different approaches in this issue [9-12].

In this study, our goal was to evaluate the importance of each BI-RADS descriptor of "enhancing masses" and its relation to benign outcome or malignant pathology among a group of patients with a final assessment of BI-RADS categories 3-5.

\section{Material and methods}

\section{Patient enrolment}

The study was approved by the ethics committee of the Tehran University of Medical Sciences. In a database of patients referred from September 2007 until March 2013, for breast MRI, we included patients with a final assessment BI-RADS categories 3, 4 and 5 who had a pathological examination of MRI-detected breast lesions. Tissue sampling included core needle/vacuum-assisted image guided biopsy before the operation, post-operative pathologic examination, or both. We excluded patients with a previous biopsyproven malignancy (BI-RADS category 6), any history of neo-adjuvant chemotherapy or those who showed a discrepancy between the pre-operative and post-operative pathology results. We included the follow-up of patients who did not have surgery, and those without follow-up MRI for at least 12 months were excluded. In addition, as we did not have access to MRI-guided biopsy in that period, we excluded patients who were referred to other centers for MRI-guided biopsy.

Finally, 433 enhancing mass lesions (in 312 patients) were enrolled in the study. Among these masses, 117 had a BI-RADS category of 3 (probably a benign finding), 263 had a BI-RADS category of 4 (suspicious lesions) and 53 had a BI-RADS category of 5 (highly suggestive of malignancy)
(Figures 1 and 2). In addition to evaluation of the recent mammography, second look ultrasound examination was obtained for all BI-RADS 4 and 5 category enhancing masses on MRI.

In case of positive ultrasound or mammographic findings that could be correlated with the MRI enhancing mass, core needle biopsy with a 14-gauge needle or vacuum-assisted biopsy with a 9- or 12-gauge needle was performed.

A small group of enhancing masses with $\mathrm{BI}$ RADS 3 category assessment that could not be localized on ultrasound examination or mammography was followed by a 6- and 12-month MRI. Also some patients with BI-RADS 4 category masses who did not undergo MRI-guided biopsy and had follow-up MRI in our institute were included.

All 53 patients with BI-RADS 5 category masses underwent image-guided biopsy, finally confirmed by surgery. The masses with a stable or downgraded BI-RADS score after 12 months were considered as benign and were scheduled for follow-up by sonography and/or screening mammography depending on the patient's age.

\section{Magnetic resonance imaging protocol}

Magnetic resonance imaging was conducted using a 1.5T Signa system (General Electric Medical Systems, USA) with a bilateral phased-array 4-channel breast coil. All patients were scanned in the prone position. Among the premenopausal patients, MRI was conducted during the second week of their menstrual cycle, whenever possible. Axial T1-weighted and axial STIR images were obtained, followed by six series of axial dynamic T1-weighted three-dimensional, fat-suppressed spoiled gradient-echo, one set prior to and five series after the rapid bolus injection of $0.1 \mathrm{mmol} / \mathrm{kg}$ gadolinium-DTPA (Dotarem, Guerbet), followed by a $15 \mathrm{ml}$ saline injection.

The axial T1-weighted sequence was obtained with the following parameters: TR/TE: 400/10; BW: $31.25 \mathrm{~Hz} /$ pixel; FOV: usually $32 \mathrm{~mm}$; slice thickness: $5.0 \mathrm{~mm}$; matrix size: $384 \times 256$; NEX: 1 . The parameters of axial STIR were as follows: TR/ TE: 4500/63; bandwidth: 62.50; FOV: usually 32; slice thickness: $5.0 \mathrm{~mm}$; matrix size: $320 \times 256$; NEX: 1. A dynamic T1-weighted three-dimensional, fat-suppressed spoiled gradient-echo sequence with the following parameters was obtained: TR/ TE: 9/4; BW: 31.25; FOV: 32; slice thickness: $4.0 \mathrm{~mm}$ with no intersection gap; matrix size: $352 \times 288$; NEX: 1; FA: 300.

The dynamic series were obtained every 60-90 s, so all six series were performed within 9 min of i.v. contrast injection. MR mammograms were interpreted by a dedicated, sub-specialized, expert radiologist with more than 15 years of experience in breast imaging. The assessment of morphologic 

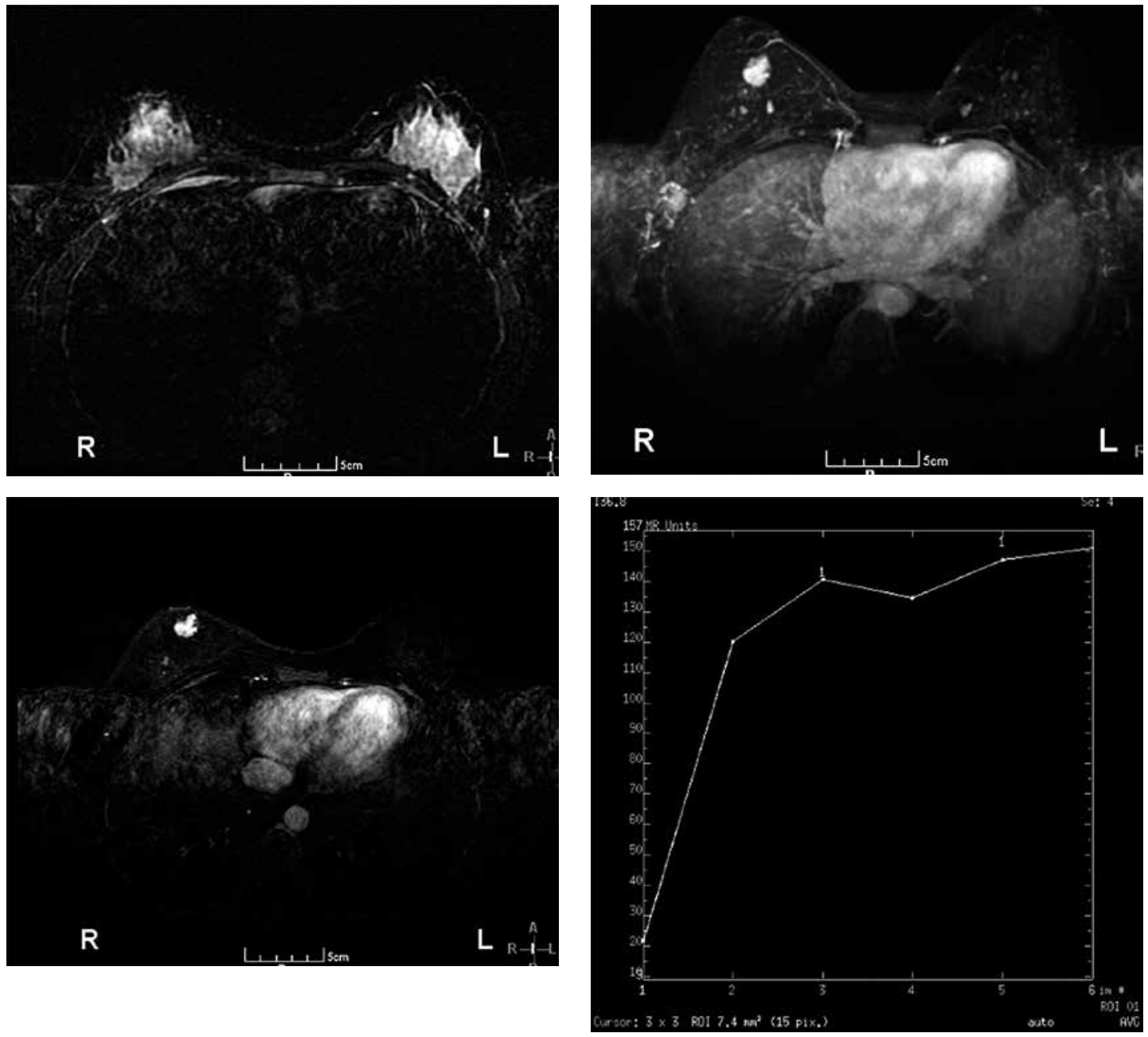

Figure 1. A 45-year-old female patient with screening detected borderline appearing mass in LIQ of right breast on ultrasound examination, in breast MRI, a low T1, low STIR, enhancing mass was detected in the right lower inner quadrant, which showed irregular shape and borders, heterogeneous internal pattern and type 2 curves assessed as BI-RADS category type 4 . The mass proved to be a complex fibroadenoma on core needle biopsy

configuration and kinetic enhancement was based on the American College of Radiology BI-RADS MRI lexicon version 4. We used CAD-STREAM for a systematized interpretation of MR mammograms and five series of three-dimensional subtracted images were processed by CAD-stream Confirma. The morphologic configurations included focus/ foci (punctuate dots of enhancement smaller than $5 \mathrm{~mm}$ ), mass (enhancing masses that had space-occupying features larger than $5 \mathrm{~mm}$ ) and non-mass-like enhancement (areas of enhancement that had neither tri-dimensional shape nor typical mass characteristics) [13]. Lesions showing slow initial contrast enhancement (less than 50\% enhancement in the first 60-90 s) were excluded, and those with $50-100 \%$ and more than $100 \%$ initial rise of the time-intensity curve were named as intermediate (non-rapid) and rapid type of curves respectively. Among three types of time-intensity curves including persistent, plateau and rapid-wash out, the most concerning curve type in each lesion was considered for interpretation. Based on morphologic and kinetic enhancement characteristic results, all patients were assigned a BI-RADS-category score of 0-6 [14].

\section{Statistical analysis}

We reviewed enhancing masses on breast MRI with BI-RADS 3, 4, and 5 assessment categories, and compared different MRI findings descriptors with final outcome of the patients. We used the $\chi^{2}$ test for assessment of statistical difference of categorical variables among malignant and benign lesions. In order to calculate diagnostic indices including sensitivity, specificity, positive predictive value, negative predictive value, positive likelihood ratio and negative likelihood ratio, we used the cross tabulations in dichotomized variables, and we also calculated the odds ratios based on these tables. In addition, multivariate logistic regression analysis was used for assessment of malignancy as the dependent variable and different 

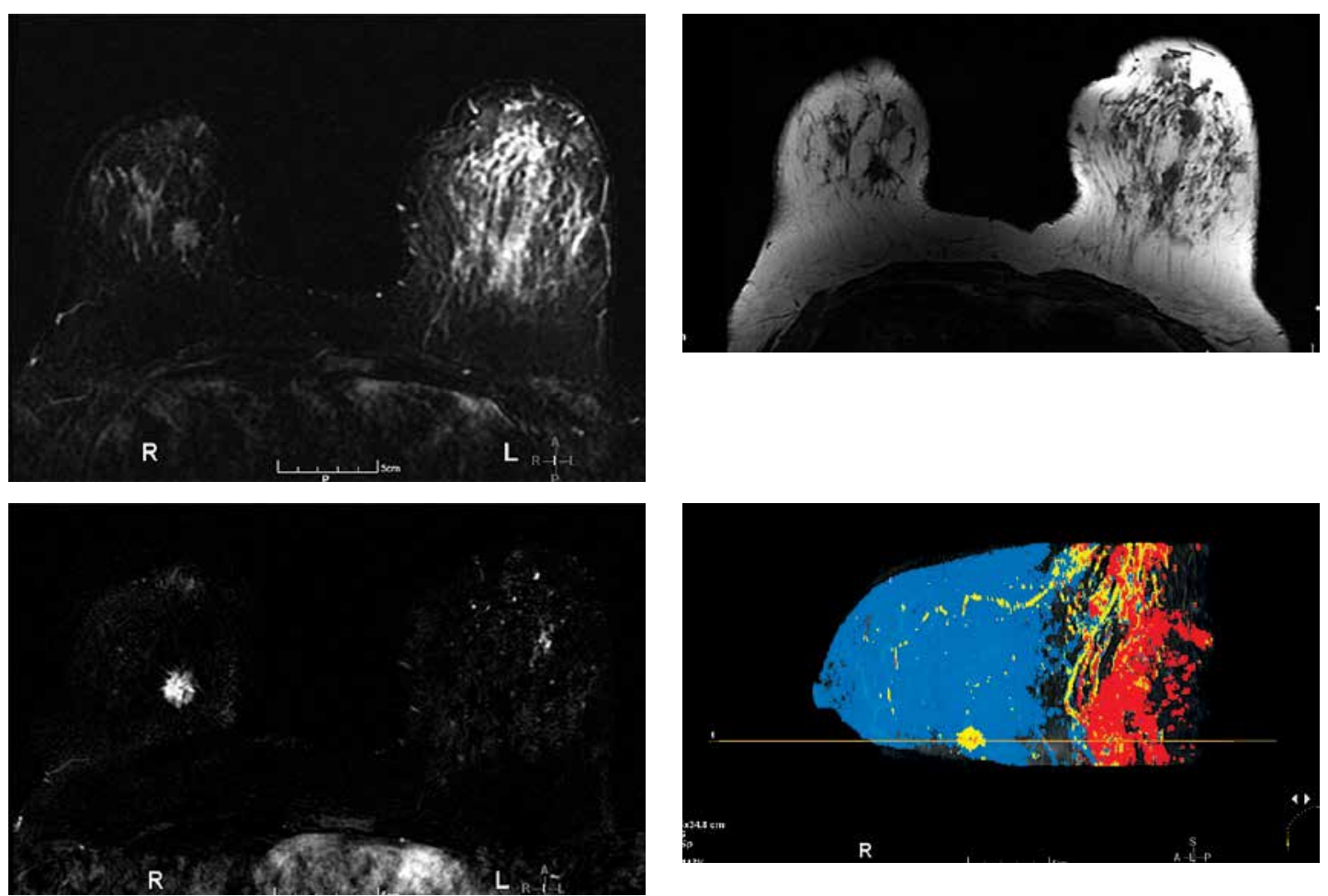

Figure 2. A 45-year-old high-risk female patient presented with a palpable mass in the right lower part, referred for preoperative staging, after suspicious mammography and ultrasound. Breast MRI on workstation as reviewed by radiologist showed: A - A round enhancing mass with speculated borders, dark on T1W, and isosignal on STIR images. B - The mass shows heterogeneous internal pattern with type 2 and type 3 dynamic curves (as yellow and red colors); final BI-RADS assessment was category 5 . The mass proved to be invasive ductal carcinoma

descriptors to find the independent MRI descriptors which could predict the malignancy.

All data were analyzed using SPSS ver. 16 for Windows (SPSS Inc., Chicago, IL, USA). A p-value $<0.05$ was accepted to be statistically significant.

\section{Results}

In a retrospective study we searched a database of pathologically proven breast lesion patients who underwent dynamic contrast-enhanced breast MRI examination before operation or biopsy, and finally 433 masses among 312 patients were evaluated. The mean age of the patients was $45.1 \pm 10.5$ years (range: 18 to 83 years). Among breast lesions, 198 (45.7\%) were positioned in the right breast and 235 (54.3\%) were detected in the left breast. The most common location was upper outer quadrant (UOQ) (21.7\%) followed by lower outer quadrant (LOQ) (20.8\%). In total, 88 (20.3\%) observed masses were malignant. The most frequent BI-RADS score was $4(n=263,60.7 \%)$, followed by $3(n=117,27 \%)$. Distribution of other imaging characteristics of the mass lesions is summarized in Table I. Keeping in mind the fact that early background enhancement may obscure some details, the reported early background enhancement was observed in $89(26.7 \%)$ of 312 patients. The most common shape of the masses was an oval/lobulated shape that was seen in 288 lesions (66.5\%) (Table I).

The mean diameter of the enhancing masses was $12.4 \pm 6.1 \mathrm{~mm}$ (range: $5-40$ ), and 117 (33.6\%) lesions had a diameter less than $10 \mathrm{~mm}$. The mean diameter of benign and malignant masses was $11.6 \pm 5.4 \mathrm{~mm}$ and $15.7 \pm 7.5 \mathrm{~mm}$, respectively $(p<0.0001)$. The association of different BI-RADS descriptors with malignancy was assessed. In our study group selected based on mentioned inclusion and exclusion criteria, masses greater than $10 \mathrm{~mm}$ were more associated with malignancy (26.4\% vs. $7.7 \%, p$-value < 0.001$)$.

The frequency of malignant and benign pathologies in each descriptor is presented in Table II. In all descriptors except for enhancement type, the distribution of malignancy was statistically different in subgroups.

In the cross tabulation, in each descriptor group, the subclass that showed the highest association with malignancy was determined. For example, among all subclasses of shape (including round, oval/lobulated and irregular shapes), the frequency of malignancy was higher in irregular masses $38 \%$ in irregular, $16.7 \%$ in oval/lobulated and $15.2 \%$ in round masses). Based on the highest association with malignancy, we categorized each descriptor into two groups, the first group with the highest 
Table I. Distribution of MRI descriptors in patients with breast mass lesions

\begin{tabular}{|c|c|c|c|}
\hline Data characteristics & Categorization & Number & Percent \\
\hline \multirow[t]{3}{*}{ BI-RADS } & 3 & 117 & 27 \\
\hline & 4 & 263 & 60.8 \\
\hline & 5 & 53 & 12.2 \\
\hline \multirow[t]{2}{*}{ Early background enhancement } & Yes & 89 & 26.7 \\
\hline & No & 244 & 73.3 \\
\hline \multicolumn{4}{|l|}{ Descriptors: } \\
\hline \multirow[t]{3}{*}{ Shape } & Round & 66 & 15.2 \\
\hline & Oval/lobulated & 288 & 66.5 \\
\hline & Irregular & 79 & 18.2 \\
\hline \multirow[t]{2}{*}{ Margin } & Circumscribed & 229 & 52.9 \\
\hline & Noncircumscribed & 204 & 47.1 \\
\hline \multirow[t]{6}{*}{ Internal enhancement } & Central enhancement & 1 & 0.2 \\
\hline & Dark internal septation & 28 & 6.5 \\
\hline & Homogeneous & 198 & 45.7 \\
\hline & Rim enhancement & 38 & 8.8 \\
\hline & Heterogeneous & 163 & 37.6 \\
\hline & $\begin{array}{c}\text { Enhanced internal } \\
\text { septation }\end{array}$ & 3 & 0.7 \\
\hline \multirow[t]{2}{*}{ Enhancement type } & Rapid & 409 & 94.5 \\
\hline & Non-rapid & 24 & 5.5 \\
\hline \multirow[t]{3}{*}{ Curve type } & Persistent & 98 & 22.6 \\
\hline & Plateau & 106 & 24.5 \\
\hline & Wash-out & 229 & 52.9 \\
\hline \multirow[t]{3}{*}{ T2 Finding } & Hypo-signal & 34 & 12 \\
\hline & Iso-signal & 63 & 22.3 \\
\hline & Hyper-signal & 186 & 65.7 \\
\hline \multirow[t]{2}{*}{ Size } & $<10 \mathrm{~mm}$ & 117 & 33.6 \\
\hline & $\geq 10 \mathrm{~mm}$ & 231 & 66.4 \\
\hline
\end{tabular}

frequency of malignancy and the second group consisting of other descriptors. For example, regarding shape, the irregular subclass was considered as the first group and the two other subclasses (including oval/lobulated and round masses) were integrated in one group as the second group. We cross-tabulated these two new groups of BI-RADS descriptors with the pathology results and calculated the odds ratio and diagnostic indices including sensitivity, specificity, positive predictive value, negative predictive value, positive likelihood ratio and negative likelihood ratio (Tables III and IV).

In a multivariate logistic regression analysis in which the pathology result was considered as the dependent variable and the above-mentioned dichotomized BI-RADS descriptors were considered as the independent variables, the Cox model $R$ square was 0.23 and the size, margin, enhancement type and curve type remained significant in the model, while the shape and internal enhancement did not have a significant $p$-value in the model (Table V).

\section{Discussion}

Magnetic resonance imaging can detect breast cancers that are occult in other imaging modalities or physical examination. The sensitivi- 
Table II. Distribution of malignant and benign features according to each descriptor and BI-RADS of MRI

\begin{tabular}{|c|c|c|c|c|}
\hline Descriptors & Categorization & Malignant, $n(\%)$ & Benign, $n(\%)$ & $P$-value \\
\hline \multirow[t]{3}{*}{ Shape } & Round & $10(15.2)$ & $56(84.8)$ & \multirow[t]{3}{*}{$<0.001$} \\
\hline & Oval/lobulated & $48(16.7)$ & $240(83.3)$ & \\
\hline & Irregular & $30(38)$ & $49(62)$ & \\
\hline \multirow[t]{2}{*}{ Margin } & Circumscribed & $18(7.9)$ & $211(92.1)$ & \multirow[t]{2}{*}{$<0.001$} \\
\hline & Non circumscribed & $70(34.3)$ & $134(65.7)$ & \\
\hline \multirow[t]{6}{*}{ Internal enhancement } & Central enhancement & $0(0)$ & $1(100)$ & \multirow[t]{6}{*}{0.041} \\
\hline & Dark internal septation & $1(3.6)$ & $27(96.4)$ & \\
\hline & Homogeneous & $35(17.7)$ & $163(82.3)$ & \\
\hline & Rim enhancement & $9(23.7)$ & $29(76.3)$ & \\
\hline & Heterogeneous & $42(25.8)$ & $121(74.2)$ & \\
\hline & Enhanced internal septation & $1(33.3)$ & $2(66.7)$ & \\
\hline \multirow[t]{2}{*}{ Enhancement type } & Non-rapid & $2(8.3)$ & $22(91.7)$ & \multirow[t]{2}{*}{0.133} \\
\hline & Rapid & $86(21)$ & $323(79)$ & \\
\hline \multirow[t]{3}{*}{ Curve type } & Persistent & $5(5.1)$ & $93(94.9)$ & \multirow[t]{3}{*}{$<0.001$} \\
\hline & Plateau & 19 (17.1) & $87(82.1)$ & \\
\hline & Wash-out & $64(27.9)$ & $165(72.1)$ & \\
\hline \multirow[t]{2}{*}{ Size } & $<10 \mathrm{~mm}$ & $9(7.7)$ & $108(92.3)$ & \multirow[t]{2}{*}{$<0.001$} \\
\hline & $\geq 10 \mathrm{~mm}$ & $61(26.4)$ & $170(73.6)$ & \\
\hline \multirow[t]{3}{*}{ BI-RADS } & 3 & $4(3.4)$ & $113(96.6)$ & \multirow[t]{3}{*}{$<0.001$} \\
\hline & 4 & $41(15.6)$ & $222(84.4)$ & \\
\hline & 5 & $43(81.1)$ & $10(18.9)$ & \\
\hline
\end{tabular}

ty of MRI for breast cancer is higher than $90 \%$ (94-99\%), while regarding overlapping features among malignant and benign lesions; its specificity is significantly lower and remains variable, leading to considerable false positive results and unnecessary invasive diagnostic procedures $[2,3$, 14-16].

Most malignant breast tumors are highly vascular and are easily discovered in contrast-enhanced MRI. In addition, there are many benign lesions that are hypervascular and may cause difficulty in interpretation of the breast MRI. Some benign lesions such as fibrocystic changes and sclerosing adenosis have been presented as misleading pathologies in recent articles [17-19]. In this study, we observed similar overlapping with a wide range of benign lesions in our false positive results, especially in the BI-RADS 4 category. Accordingly, any improvement in the imaging methods and interpretation leading to higher specificity of breast MRI without sacrificing the sensitivity would be a considerable achievement. Since in the MRI BI-RADS reporting system, the radiologist is dealing with multiple morphologic and kinetic descriptors, defining the relative importance of each descriptor seems to be useful for this purpose.

In this article, we assessed the odds ratio (OR) and also diagnostic indices of each MRI descriptor in enhancing breast masses. Based on our proposed grouping for descriptors, the highest odds ratio was seen for margin, followed by the dynamic curve type, mass size, and shape.

On the other hand, we assessed diagnostic indices of each descriptor individually in malignant breast masses that showed high sensitivity and low specificity in the type of enhancement, curve type, and size, while this analysis showed high specificity (fewer unnecessary procedures) and a low sensitivity for shape and margin. In addition, for internal enhancement, both sensitivity and specificity were in a medium range. These findings mean lower false positive results could be achieved by considering morphologic indices, while lower false negative results are obtained in dynamic curves and lesion size.

Some other studies have focused on the rate of false positive and false negative results of the 
Table III. Odds ratio for differentiating benign and malignant features of breast masses in each MRI descriptor

\begin{tabular}{|c|c|c|c|c|c|}
\hline Descriptors & Categorization & Malignant, $n$ (\%) & Benign, $n(\%)$ & $P$-value & OR $(95 \% \mathrm{Cl})$ \\
\hline \multirow[t]{2}{*}{ Shape } & Irregular & $30(38)$ & $49(62)$ & $<0.001$ & $3.1(1.8-5.3)$ \\
\hline & Other & $58(16.4)$ & $296(83.6)$ & & \\
\hline \multirow[t]{2}{*}{ Margin } & $\begin{array}{l}\text { Non circumscribed } \\
\text { (only spiculated) }\end{array}$ & $40(60.6)$ & $26(39.4)$ & $<0.001$ & $10.2(5.7-18.3)$ \\
\hline & $\begin{array}{l}\text { Circumscribed and other } \\
\text { non circumscribed }\end{array}$ & $48(13.1)$ & 319 (86.9) & & \\
\hline \multirow[t]{2}{*}{$\begin{array}{l}\text { Internal } \\
\text { enhancement }\end{array}$} & $\begin{array}{l}\text { Heterogeneous/rim/ } \\
\text { enhancing septa }\end{array}$ & $52(25.5)$ & $152(74.5)$ & 0.013 & $1.8(1.1-2.9)$ \\
\hline & Other & $36(15.9)$ & $191(84.1)$ & & \\
\hline \multirow{2}{*}{$\begin{array}{l}\text { Enhancement } \\
\text { type }\end{array}$} & Rapid & $86(21)$ & $323(79)$ & 0.133 & $2.9(0.7-12.7)$ \\
\hline & Non-rapid & $2(8.3)$ & $22(91.7)$ & & \\
\hline \multirow[t]{2}{*}{ Curve type } & Wash-out or plateau & $83(24.8)$ & $252(75.2)$ & $<0.001$ & $6.1(2.4-15.6)$ \\
\hline & Persistent & $5(5.1)$ & $93(94.9)$ & & \\
\hline \multirow[t]{2}{*}{ Size } & $\geq 10 \mathrm{~mm}$ & $61(26.4)$ & $170(73.6)$ & $<0.001$ & $4.3(2.1-9)$ \\
\hline & $<10 \mathrm{~mm}$ & $9(7.7)$ & $108(92.3)$ & & \\
\hline
\end{tabular}

Table IV. Diagnostic indices of the BI-RADS descriptors for malignancy

\begin{tabular}{|c|c|c|c|c|c|c|c|c|c|c|}
\hline Descriptor & TP & FN & TN & FP & $\begin{array}{c}\text { Sen. } \\
(95 \% \mathrm{Cl})\end{array}$ & $\begin{array}{c}\text { Spec. } \\
(95 \% \mathrm{Cl})\end{array}$ & $\begin{array}{c}\text { PPV } \\
(95 \% \mathrm{Cl})\end{array}$ & $\begin{array}{c}\text { NPV } \\
(95 \% \mathrm{Cl})\end{array}$ & $\begin{array}{c}\text { PLR } \\
(95 \% \mathrm{Cl})\end{array}$ & $\begin{array}{c}\text { NLR } \\
(95 \% \mathrm{Cl})\end{array}$ \\
\hline $\begin{array}{l}\text { Size } \\
(\geq 10 \mathrm{~mm})\end{array}$ & 61 & 9 & 108 & 170 & $\begin{array}{c}87 \\
(77-94)\end{array}$ & $\begin{array}{c}39 \\
(33-45)\end{array}$ & $\begin{array}{c}26 \\
(21-33)\end{array}$ & $\begin{array}{c}92 \\
(86-96)\end{array}$ & $\begin{array}{c}1.4 \\
(1.3-1.6)\end{array}$ & $\begin{array}{c}3 \\
(1.6-5.7)\end{array}$ \\
\hline $\begin{array}{l}\text { Shape } \\
\text { (irregular) }\end{array}$ & 30 & 58 & 296 & 49 & $\begin{array}{c}34 \\
(24-45)\end{array}$ & $\begin{array}{c}86 \\
(82-89)\end{array}$ & $\begin{array}{c}38 \\
(27-50)\end{array}$ & $\begin{array}{c}84 \\
(79-87)\end{array}$ & $\begin{array}{c}2.4 \\
(1.6-3.5)\end{array}$ & $\begin{array}{c}1.3 \\
(1.1-1.5)\end{array}$ \\
\hline $\begin{array}{l}\text { Margin } \\
\text { (spiculated non- } \\
\text { circumscribed) }\end{array}$ & 40 & 48 & 319 & 26 & $\begin{array}{c}45 \\
(35-56)\end{array}$ & $\begin{array}{c}92 \\
(89-95)\end{array}$ & $\begin{array}{c}61 \\
(48-72)\end{array}$ & $\begin{array}{c}87 \\
(83-90)\end{array}$ & $\begin{array}{c}6 \\
(3.9-9.3)\end{array}$ & $\begin{array}{c}1.7 \\
(1.4-2.1)\end{array}$ \\
\hline $\begin{array}{l}\text { Internal enhancement } \\
\text { (heterogeneous /rim/ } \\
\text { enhancing septa) }\end{array}$ & 52 & 36 & 191 & 152 & $\begin{array}{c}59 \\
(48-69)\end{array}$ & $\begin{array}{c}56 \\
(50-61)\end{array}$ & $\begin{array}{c}25 \\
(20-32)\end{array}$ & $\begin{array}{c}84 \\
(79-89)\end{array}$ & $\begin{array}{c}1.3 \\
(1.1-1.6)\end{array}$ & $\begin{array}{c}1.4 \\
(1-1.8)\end{array}$ \\
\hline $\begin{array}{l}\text { Enhancement type } \\
\text { (rapid) }\end{array}$ & 86 & 2 & 22 & 323 & $\begin{array}{c}98 \\
(92-99)\end{array}$ & $\begin{array}{c}6 \\
(4-10)\end{array}$ & $\begin{array}{c}21 \\
(17-25)\end{array}$ & $\begin{array}{c}92 \\
(73-99)\end{array}$ & $\begin{array}{c}1.04 \\
(1-1.09)\end{array}$ & $\begin{array}{c}2.8 \\
(0.67- \\
11.7)\end{array}$ \\
\hline $\begin{array}{l}\text { Curve type } \\
\text { (Wash-out and plateau) }\end{array}$ & 83 & 5 & 93 & 252 & $\begin{array}{c}94 \\
(87-98) \\
\end{array}$ & $\begin{array}{c}27 \\
(22-32) \\
\end{array}$ & $\begin{array}{c}25 \\
(20-30) \\
\end{array}$ & $\begin{array}{c}95 \\
(88-98) \\
\end{array}$ & $\begin{array}{c}1.3 \\
(1.2-1.4) \\
\end{array}$ & $\begin{array}{c}4.7 \\
(2-11.3) \\
\end{array}$ \\
\hline BI-RADS $(4,5)$ & 84 & 4 & 113 & 232 & $\begin{array}{c}95 \\
(89-99)\end{array}$ & $\begin{array}{c}33 \\
(28-38)\end{array}$ & $\begin{array}{c}27 \\
(22-32)\end{array}$ & $\begin{array}{c}97 \\
(91-99)\end{array}$ & $\begin{array}{c}1.4 \\
(1.3-1.5)\end{array}$ & $\begin{array}{c}7.2 \\
(2.7-19)\end{array}$ \\
\hline
\end{tabular}

NLR - neutrophil to lymphocyte ratio, PPV - positive predictive value.

Table V. Multivariate logistic regression model for estimating malignancy according to BI-RADS descriptors

\begin{tabular}{|lccccc|}
\hline Variable & $B$ & Sig. & Exp (B) & \multicolumn{2}{c|}{$95 \%$ CI for Exp (B) } \\
\cline { 5 - 6 } & & & & Lower & Upper \\
\hline Size $(\geq 10 \mathrm{~mm})$ & 1.1 & 0.012 & 2.9 & 1.3 & 6.8 \\
\hline Shape (irregular) & 0.61 & 0.11 & 1.8 & 0.87 & 3.9 \\
\hline Margin (spiculated non-circumscribed) & 2.6 & $<0.0001$ & 13.0 & 5.4 & 31.5 \\
\hline $\begin{array}{l}\text { Internal enhancement heterogeneous/ } \\
\text { rim/enhancing septa }\end{array}$ & 0.15 & 0.65 & 1.2 & 0.6 & 2.3 \\
\hline Enhancement type (rapid) & 2.5 & 0.03 & 12.1 & 1.3 & 116.1 \\
\hline Curve type (Wash-out and plateau) & 2.6 & 0.002 & 14.0 & 2.7 & 72.9 \\
\hline Constant & -14.845 & $<0.0001$ & 0.000 & & \\
\hline
\end{tabular}


MRI BI-RADS final assessment. They have also assessed the relative importance of each descriptor in MRI BI-RADS. Mahoney et al. reported $29 \%$ of their BI-RADS 5 and $79 \%$ of their BI-RADS 4 lesions as benign pathologies positive predictive value (PPV) equal to $71 \%$ and $21 \%$, respectively) [9]. In another study, $33 \%$ and $81 \%$ of BI-RADS 5 and 4 lesions were benign (PPV equal to $67 \%$ and $19 \%$, respectively) [20]. In our series, $18.9 \%$ of BI-RADS 5 and $84.4 \%$ of BI-RADS 4 lesions were benign (PPV equal to $81.1 \%$ and $15.6 \%$, respectively).

As observed in different studies, the rate of false positive cases is considerable, especially in BI-RADS 4 lesions. This means that the current policy of breast lesion management has been adjusted based on a conservative set point to decrease the cancer-missing rate at the cost of performing unnecessary invasive procedures.

In the review of the literature, we found some studies focusing on the efficacy of each descriptor of breast MRI [9-12, 21-26]. In spite of somewhat different designs and settings, the general goal of these studies is to assess the relative importance of each descriptor in the diagnosis of malignant breast lesions.

In an overall view, our results showed a good sensitivity (and thus a low cancer-missing rate) for size, enhancement type and curve type of kinetic assessment, while it showed a good specificity (and thus a decreased false positive rate leading to fewer unnecessary invasive procedures) among patients for morphologic descriptors of shape and margin. In addition, the best OR was seen for margin and curve type, then for size and shape, and other descriptors showed lower ORs. Findings of other studies were quite similar to our study; however, there were different results among other studies. These differences were related to stronger or weaker diagnostic indices in different descriptors; as mentioned, these differences are partly related to inhomogeneous settings and patient enrollment settings among these studies. Gutierrez et al. reported ORs of 2.8-4.6 for size, shape, margin and internal enhancement in diagnosis of malignancy [20]. However, they considered only BI-RADS 4 and 5 lesions. This could lower the indices, as the most lesions that lower diagnostic indices are BI-RADS 4 lesions, and excluding BI-RADS 3 lesions could decrease the diagnostic indices and ORs. The positive likelihood ratio of morphologic characteristics (including irregular shape, spiculated margin and heterogeneous internal enhancement) in the study of Baltzer et al. was similar to our study [10]. Mahoney et al. reported that among 121 enhancing masses, irregular shape, spiculated and irregular margins and rapid internal enhancement were most predictive of malignancy [9]. In the study of Wedegärtner et al., irregular lesion contour (margin) had the best efficacy in determining malignancy (sensitivity and specificity of $83 \%$ and $76 \%$, respectively) [12]. Thus, compared to our findings, in somewhat lower specificity, they obtained a significantly better sensitivity for margin. Considerable power of spiculated margin presence for malignancy was reported by Schnall et al. [11] In addition, they stated that kinetic characteristics are among the most reliable criteria suggestive for malignancy. Tozaki et al. reported a spiculated margin to be suggestive of malignancy in all masses and an irregular shape to be related to malignancy in $97 \%$ of cases [21].

To find independent MRI descriptors that could predict malignancy, we performed a multivariate analysis, in which margin, size and kinetic characteristics were statistically significant variables. Similarly, Gutierrez et al. found shape as a less significant descriptor of BI-RADS in the multivariate model [20]. Although more powerful variables such as "margin" do not exclude the less powerful descriptors such as "shape" from the multivariate model in the current study, based on our results, neither morphology nor kinetic characteristics have been completely excluded from the model in our study. We can conclude that both morphologic and kinetic descriptors bear a valid weight in the diagnosis of masses and neither could be ignored.

Finally, the new BI-RADS lexicon seems to be more efficient in discrimination of malignant and benign lesions and may increase sensitivity while not sacrificing specificity, and as a result reduces unnecessary biopsy and patient anxiety. Future studies should compare the results.

In conclusion, it seems that morphologic descriptors of MRI BI-RADS are highly specific while kinetic related descriptors are highly sensitive in diagnosis of malignant masses of the breast. Appropriate consideration and combination of different BI-RADS findings could help in better interpretation of MRI BI-RADS and lowering the rate of false reports.

\section{Conflict of interest}

The authors declare no conflict of interest.

\section{References}

1. Mann RM, Kuhl CK, Kinkel K. Breast MRI: guidelines from the European Society of Breast Imaging. Eur Radiol 2008; 18: 1307-18.

2. Sardanelli F, Giuseppetti GM, Canavese G, Cataliotti L, Corcione S. Indications for breast magnetic resonance imaging. Consensus document "Attualità in senologia", Florence 2007. Radiol Med 2008; 113: 1085-95.

3. Martincich L, Montemurro F, Cirillo S, Marra V, De Rosa G. Role of magnetic resonance imaging in the prediction of tumor response in patients with locally advanced breast 
cancer receiving neoadjuvant chemo-therapy. Radiol Med 2003; 106: 51-8.

4. Londero V, Bazzocchi M, Del Frate C, et al. Locally advanced breast cancer: comparison of mammography, sonography and MR imaging in evaluation of residual disease in women receiving neoadjuvant chemotherapy. Eur Radiol 2004; 14: 1371-9.

5. Thukral A, Thomasson DM, Chow CK, et al. Inflammatory breast cancer: dynamic contrast-enhanced MR in patients receiving bevacizumab - initial experience. $\mathrm{Ra}$ diology 2007; 244: 727-35.

6. Zakhireh J, Gomez R, Esserman L. Converting evidence to practice: a guide for the clinical application of MRI for the screening and management of breast cancer. Eur J Cancer 2008; 44: 2742-52.

7. Rankin SC. MRI of the breast. Br J Radiol 2000; 73: 806-18.

8. Uematsu T, Kasami M. MR imaging findings of benign and malignant circumscribed breast masses: part 2. Cystic circumscribed masses. Jpn J Radiol 2009; 27: 405-9.

9. Mahoney MC, Gatsonis C, Hanna L, DeMartini WB, Lehman C. Positive predictive value of BI-RADS MR imaging. Radiology 2012; 264: 51-8.

10. Baltzer PA, Benndorf M, Dietzel M, Gajda M, Runnebaum IB. False-positive findings at contrast-enhanced breast MRI: a BI-RADS descriptor study. AJR Am J Roentgenol 2010; 194: 1658-63.

11. Schnall MD, Blume J, Bluemke DA, DeAngelis GA, DeBruhl N. Diagnostic architectural and dynamic features at breast MR imaging: multicenter study. Radiology 2006; 238: 42-53

12. Wedegärtner $U$, Bick $U$, Wörtler K, Rummeny E, Bongartz G. Differentiation between benign and malignant findings on MR-mammography: usefulness of morphologicalcriteria. Eur Radiol 2001; 11: 1645-50.

13. Heywang-Köbrunner SH, Viehweg P, Heinig A, Küchler C. Contrast-enhanced MRI of the breast: accuracy, value, controversies, solutions. Eur J Radiol 1997; 24: 94-108.

14. Mann RM, Kuhl CK, Kinkel K, Boetes C. Breast MRI: guidelines from the European Society of Breast Imaging. Eur Radiol 2008; 18: 1307-18.

15. Schnall MD, Ikeda DM. Lesion Diagnosis Working Group report. J Magn Reson Imaging 1999; 10: 982-90.

16. American College of Radiology. Breast Imaging Reporting and Data System Atlas (BI-RADS Atlas). Reston, VA: American College of Radiology 2003.

17. Chen JH, Nalcioglu O, Su MY. Fibrocystic change of the breast presenting as a focal lesion mimicking breast cancer in MR imaging. J Magn Reson Imaging 2008; 28: 1499-505.

18. Oztekin PS, Tuncbilek I, Kosar P, Gültekin S, Oztürk F. Nodular sclerosing adenosis mimicking malignancy in the breast: magnetic resonance imaging findings. Breast J 2011; 17: 95-7.

19. Liberman L, Morris EA, Lee MJ, et al. Breast lesions detected on MR imaging: features and positive predictive value. AJR Am J Roentgenol 2002; 179: 171-8.

20. Gutierrez RL, DeMartini WB, Eby PR, et al. BI-ADS lesion characteristics predict likelihood of malignancy in breast MRI for masses but not for non mass like enhancement. AJR Am J Roentgenol 2009; 193: 994-1000.

21. Tozaki M, Igarashi T, Fukuda K. Positive and negative predictive values of BI-RADS-MRI descriptors for focal breast masses. Magn Reson Med Sci 2006; 5: 7-15.

22. Cho YH, Cho KR, Park EK, Kyoung Seo B, Woo OH. Significance of additional non-mass enhancement in patients with breast cancer on preoperative $3 T$ dynamic contrast enhanced MRI of the breast. Iran J Radiol 2016; 13: e30909.

23. Gity M, Ghazi Moghadam K, Jalali AH, Shakiba M. Association of different MRI BIRADS descriptors with malignancy in non-mass-like breast lesions. Iran Red Crescent Med J 2014; 16: e26040.

24. Chen M, Zhan WW, Wang WP. Cystic breast lesions by conventional ultrasonography: sonographic subtype-pathologic correlation and BI-RADS Assessment. Arch Med Sci 2014; 10: 76-83.

25. Sadr SSH, Javanbakht J, Norouzi Javidan A, Ghaffarpour $M$, Khamse S. Descriptive epidemiology: prevalence, incidence, sociodemographic factors, socioeconomic domains, and quality of life of epilepsy: an update and systematic review. Arch Med Sci 2018; 14: 717-24.

26. Rzymski P, Wysocki PJ, Kycler W, Opala T. Correlation between insulin resistance and breast elasticity heterogeneity measured by shear wave elastography in premenopausal women - a pilot study. Arch Med Sci 2011; 7: 1017-22. 